\title{
Poly-Cauchy Numbers of the Second Kind - the Combinatorics Behind
}

\author{
Beáta Bényi ${ }^{\dagger}$ and José L. Ramírez \\ $\dagger$ Faculty of Water Sciences, University of Public Service, Baja, Hungary \\ Email: benyi.beata@uni-nke.hu \\ $¥$ Departamento de Matemáticas, Universidad Nacional de Colombia, Bogotá, Colombia \\ Email: jlramirezr@unal.edu.co
}

Received: May 7, 2021, Accepted: June 6, 2021, Published: June 18, 2021

The authors: Released under the CC BY-ND license (International 4.0)

Abstract: We introduce poly-Cauchy permutations of the second kind that are enumerated by the polyCauchy numbers of the second kind. We provide combinatorial proofs for several identities involving polyCauchy numbers of the second kind and some of their generalizations. The aim of this work is to demonstrate the power and beauty of the elementary combinatorial approach.

Keywords: Permutations; Poly-Bernoulli numbers; Poly-Cauchy numbers

2020 Mathematics Subject Classification: 05A05; 05A19

\section{Introduction}

Poly-Cauchy numbers were defined by Komatsu [14] motivated by the interesting properties of poly-Bernoulli numbers. While since the introduction many combinatorial interpretations and investigations have appeared in the literature about poly-Bernoulli numbers, there is no such direction about the poly-Cauchy numbers yet.

Let $n$ and $k$ be integers with $n \geq 0$ and $k \geq 1$. The poly-Cauchy numbers of the first kind are defined by

$$
c_{n}^{(k)}=n ! \underbrace{\int_{0}^{1} \cdots \int_{0}^{1}}_{k}\left(\begin{array}{c}
t_{1} t_{2} \cdots t_{k} \\
n
\end{array}\right) d t_{1} d t_{2} \cdots d t_{k} .
$$

Moreover, the exponential generating function of $c_{n}^{(k)}$ is given by

$$
\operatorname{Lif}_{k}(\ln (1+t))=\sum_{n=0}^{\infty} c_{n}^{(k)} \frac{t^{n}}{n !}
$$

where $\operatorname{Lif}_{k}(t)=\sum_{n=0}^{\infty} \frac{t^{n}}{n !(n+1)^{k}}$ is the $k$-th polylogarithm factorial function. Similarly, the poly-Cauchy numbers of the second kind are defined by

$$
\widehat{c}_{n}^{(k)}=n ! \underbrace{\int_{0}^{1} \cdots \int_{0}^{1}}_{k}\left(\begin{array}{c}
-t_{1} t_{2} \cdots t_{k} \\
n
\end{array}\right) d t_{1} \cdots d t_{k},
$$

and the exponential generating function of $\widehat{c}_{n}^{(k)}$ is given by

$$
\operatorname{Lif}_{k}(-\ln (1+t))=\sum_{n=0}^{\infty} \widehat{c}_{n}^{(k)} \frac{t^{n}}{n !}, \quad(k \in \mathbb{Z}) .
$$

Notice that the above generating functions have a meaning as a formal power series even if $k$ is non-positive. Therefore, the poly-Cauchy numbers of both kinds can be defined also for negative $k$. Poly-Cauchy numbers of the first kind have an explicit formula

$$
c_{n}^{(k)}=(-1)^{n} \sum_{m=0}^{n}\left[\begin{array}{l}
n \\
m
\end{array}\right] \frac{(-1)^{m}}{(m+1)^{k}},
$$


where $\left[\begin{array}{c}n \\ m\end{array}\right]$ denotes the unsigned Stirling numbers of the first kind that counts the number of permutations of $[n]:=\{1,2, \ldots, n\}$ into $m$ non-empty cycles, and can algebraically defined for instance by the following identity:

$$
\sum_{m=0}^{n}\left[\begin{array}{l}
n \\
m
\end{array}\right] x^{m}=x(x+1) \cdots(x+n-1) .
$$

Poly-Cauchy numbers of the second kind have an explicit formula

$$
\widehat{c}_{n}^{(k)}=(-1)^{n} \sum_{m=0}^{n}\left[\begin{array}{l}
n \\
m
\end{array}\right] \frac{1}{(m+1)^{k}} .
$$

For more properties about poly-Cauchy numbers of the first and the second kind see for example [8,13-15].

In the literature, along with the theory of poly-Bernoulli numbers and polynomials, the (analytical) theory of poly-Cauchy numbers have been also developed, including several identities and generalizations (polynomials, $q$-parameters), etc.

We are interested from the combinatorial point of view in poly-Cauchy numbers of the second kind with negative $k$-indices, which are integers. However, one notices that we have negative and positive integers as well, so actually $(-1)^{n} \widehat{c}_{n}^{(-k)}$ are positive integers. More precisely, we want to treat with the positive integers. For the sake of this purpose, we denote these positive integers by $\widehat{c}_{n, k}:=(-1)^{n} \widehat{c}_{n}^{(-k)}$.

Table 1 shows the first few values of the sequence $\widehat{c}_{n, k}$.

\begin{tabular}{|c||c|c|c|c|c|c|}
\hline$n \backslash k$ & 0 & 1 & 2 & 3 & 4 & 5 \\
\hline \hline 0 & 1 & 1 & 1 & 1 & 1 & 1 \\
\hline 1 & 1 & 2 & 4 & 8 & 16 & 32 \\
\hline 2 & 2 & 5 & 13 & 35 & 97 & 275 \\
\hline 3 & 6 & 17 & 51 & 161 & 531 & 1817 \\
\hline 4 & 24 & 74 & 244 & 854 & 3148 & 12134 \\
\hline
\end{tabular}

Table 1: The first values of the sequence $\widehat{c}_{n, k}$.

\section{Poly-Cauchy permutations of the second kind}

In this section, we introduce a new family of permutations enumerated by the sequence $\widehat{c}_{n, k}$. First, we recall some well-known facts about permutations. Permutations can be defined and seen from a different point of view, as a map, as an arrangement, as a product of cycles, etc. It is useful to switch between these points of view, however, it is always important to be conscious of the particular approach that is used in an argument. In our case cycles will play a crucial role.

Any permutation is the product of cycles, and cycles can be recorded uniquely: we write the largest element of each cycle first, then we arrange the cycles in increasing order of their first elements. This representation is called canonical cycle notation.

On the other hand, considering a permutation as a map, $\pi:[n] \rightarrow[n], \pi_{i}=\pi(i)$ each permutation can be written in one-line notation, $\pi=\pi_{1} \pi_{2} \cdots \pi_{n}$. There is a well-known bijection between permutations of $[n]$ written in cycle notation and written in one-line-notation, which we recall now. We say that $\pi_{i}$ is a left-to-right maximum if, for all $k<i$, we have $\pi_{k}<\pi_{i}$. For example, the permutation 3261457 has three left-to-right maxima. These are entries 3,6 , and 7 . Let $\pi$ be a permutation of $n$ elements written in canonical cycle notation, and let $\pi^{\prime}$ be the permutation written in the one-line notation that is obtained from $\pi$ by omitting all parentheses. Notice that the canonical cycle notation can be obtained from the left-to-right maxima of the permutation.

Definition 2.1. Let $n$ and $k$ be non negative integers. $A(n, k)$-poly-Cauchy permutation of the second kind is a permutation of $[n+k]$ with the following properties:

(A1) each maximal contiguous substring whose support belongs to $\{n+1, n+2, \ldots, n+k\}$ is increasing,

(A2) the left-to-right maxima of the maximal contiguous substrings whose support belongs to $\{1,2, \ldots, n\}$ are increasing from left to right, when "reading" the whole permutation.

Example 2.1. A $(9,6)$-poly-Cauchy permutation of the second kind is

$$
\pi=11-13-6-1-2-15-7-4-8-10-12-14-9-5-3 .
$$


We introduce now some terminology and notation. Let $\mathcal{P}_{n, k}$ denote the set of all $(n, k)$-poly-Cauchy permutations of the second kind. For simplicity of notation we use the notation of underlined (red) elements for $\{\underline{1}, \underline{2}, \ldots, \underline{n}\}$ and the notation of overlined (blue) elements for $\{\overline{1}, \overline{2}, \ldots, \bar{k}\}$ instead of the set $\{n+1, n+2, \ldots, n+$ $k\}$ (which is essentially the same). Given an $(n, k)$-poly-Cauchy permutation of the second kind $\pi \in \mathcal{P}_{n, k}$ we will denote the arrangement of the elements $\{\underline{1}, \ldots, \underline{n}\}$, seeing itself as a permutation of $[n]$ as $\underline{\pi^{n}}$. Similarly, we denote the permutation of the elements $\{\overline{1}, \ldots, \bar{k}\}$ as $\overline{\pi^{k}}$. Since we consider in this paper only poly-Cauchy permutation of the second kind, for the sake of simplicity we will use sometimes the expression poly-Cauchy permutation instead.

Example 2.2. The permutation given in Example 2.1 is in this notation $\overline{24} \underline{612} \overline{6} 748 \overline{135} \underline{953}$. In the first permutation the overlined (blue) sequences are $\overline{24}, \overline{6}, \overline{135}$, and the underlined (red) sequences are $\underline{612}, 748, \underline{953}$. In the overlined (blue) sequences the elements are increasingly ordered and the bold elements in the underlined (red) sequences denote the left-to-right maxima $\underline{\pi^{n}}=\underline{612748953}$ and $\overline{\pi^{k}}=\overline{246135}$.

Note that the merging $\frac{\pi^{n}}{}$ and $\overline{\pi^{k}}$ happens so that an overlined (blue) sequence is always inserted before a left-to-right maxima of $\underline{\pi^{n}}$. On the other hand, an underlined (red) sequence is always inserted after a descent top of $\overline{\pi^{k}}$. (A descent top of a permutation $\sigma$ is $\sigma_{i}$, if $\sigma_{i}>\sigma_{i+1}$, i.e., an element followed by a smaller element.)

As a visualization of a poly-Cauchy permutation of the second kind, we can use permutation matrices. Recall that for a permutation $\pi=\pi_{1} \pi_{2} \cdots \pi_{n}$, its permutation matrix is obtained by placing a dot in columns $i$ and row $\pi_{i}$ from below. The poly-Cauchy permutations of the second kind can be represented by a permutation matrix as in Figure 1.

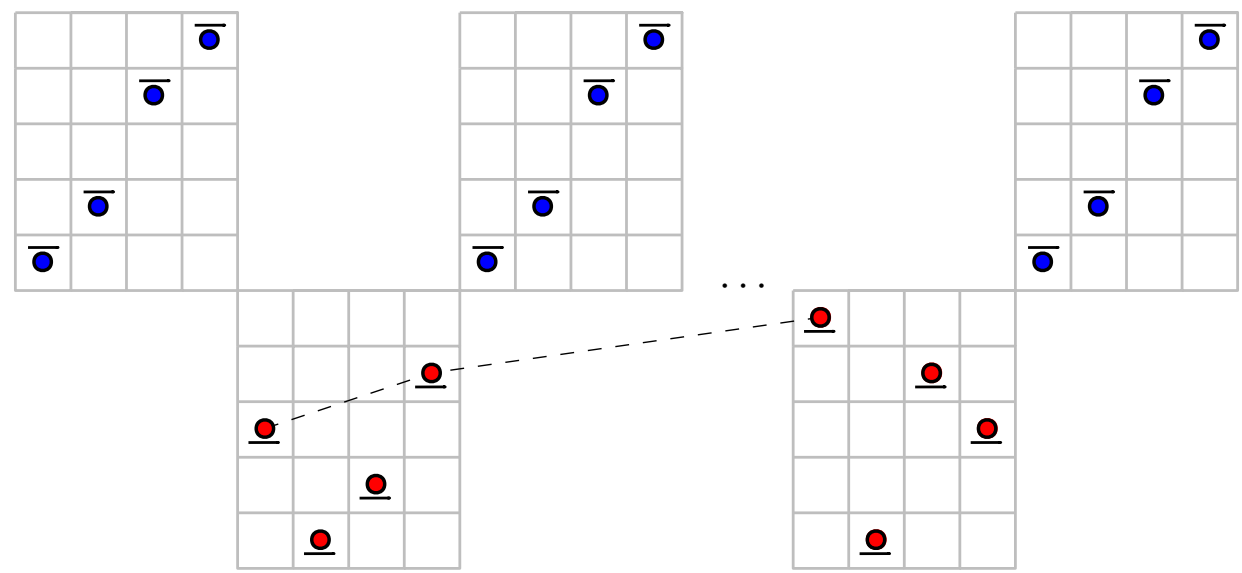

Figure 1: Decomposition of a poly-Cauchy permutation of the second kind.

Example 2.3. For $n=2$ and $k=1$ we have the 5 permutations:

$\overline{1} \underline{21}, \quad \overline{1} \underline{12}, \quad \underline{21} \overline{1}, \quad \underline{12} \overline{1}, \quad \underline{1} \overline{1} \underline{2}$.

For $n=2$ and $k=2$ we have the 13 permutations:

$$
\begin{aligned}
& \overline{12} \underline{12}, \quad \overline{12} \underline{21}, \quad \overline{1} \underline{12} \overline{2}, \quad \overline{1} \underline{21} \overline{2}, \quad \overline{2} \underline{12} \overline{1}, \quad \overline{2} \underline{11} \overline{1}, \quad \overline{1} \underline{1} \overline{2} \underline{2}, \quad \overline{2} \underline{1} \overline{1} \underline{2} \\
& \underline{12} \overline{12}, \quad \underline{21} \overline{12}, \quad \underline{1} \overline{12} \underline{2}, \quad \underline{1} \overline{1} \underline{2}, \quad \underline{1} \overline{2} \underline{2} \overline{1} \text {. }
\end{aligned}
$$

Theorem 2.1. The $(n, k)$-poly-Cauchy permutations of the second kind are enumerated by the sequence $\widehat{c}_{n, k}$.

Proof. The $(n, k)$-poly-Cauchy permutations of the second kind can be constructed by the following procedure:

1. Firstly, permute the elements $\{\underline{1}, \underline{2}, \ldots, \underline{n}\}$, and write it into canonical cycle notation. This step will ensure the condition (A2).

2. Secondly, insert the elements of $\{\overline{1}, \ldots, \bar{k}\}$ into the cycles (before or after). Notice that we have $m+1$ spaces and some of them may be left empty. The elements in the cycles are written in increasing order. This step will ensure the condition (A1).

This way we obtain an alternating sequence of cycles of $\{\underline{1}, \ldots, \underline{n}\}$ and blocks of $\{\overline{1}, \ldots, \bar{k}\}$.

It is clear that the number of $(n, k)$-poly-Cauchy permutations of the second kind constructed by this procedure is given by

$$
\widehat{c}_{n, k}=\sum_{m=0}^{n}\left[\begin{array}{l}
n \\
m
\end{array}\right](m+1)^{k} .
$$


In Figure 2 we give an example of this procedure for a $(9,6)$-poly-Cauchy permutation of the second kind.

$$
\sqcup \underline{612} \sqcup \underline{74} \sqcup \underline{8} \sqcup \underline{953} \sqcup \rightarrow \emptyset \underline{612} \emptyset \underline{74} \overline{24} \underline{8} \overline{135} \underline{953} \overline{6} \rightarrow \underline{61274} \underline{24} \underline{8} \overline{135} \underline{953 \overline{6}}
$$

Figure 2: Procedure to construct a poly-Cauchy permutation of the second kind.

We give some examples for some particular values of $n$ and $k$.

Example 2.4. For $k=0$ we have $\widehat{c}_{n, 0}=n$ !, since $(n, 0)$-poly-Cauchy permutation is simply a permutation of $[n]$.

Example 2.5. For $n=1$ we have $\widehat{c}_{1, k}=2^{k}$. To obtain a $(1, k)$-poly-Cauchy permutation we insert 1 in two sets of the overlined (blue) elements (before or after), $\{\overline{1}, \overline{2}, \ldots, \bar{k}\}$. So, $\left|\mathcal{P}_{1, k}\right|$ is the number of choosing $i$ elements from $\{\overline{1}, \overline{2}, \ldots, \bar{k}\}$ that are to the left of $\underline{1}$, allowing $i$ to be 0 and $k$.

Example 2.6. For $n=2$ we have $\widehat{c}_{2, k}=2^{k}+3^{k}$. In this case, we have the sets $\{\underline{1}, \underline{2}\}$ and $\{\overline{1}, \overline{2}, \ldots, \bar{k}\}$, so the number of ways we get such a permutation is to insert the string 21 in two sets of the overlined (blue) elements, before or after, so we have $2^{k}$ ways. We can also insert the strings 1 and 2 in three sets (possibly empty), so we have $3^{k}$ ways.

In the Theorems 2.2-2.9 we will give combinatorial arguments of some identities involving poly-Cauchy numbers, poly-Bernoulli numbers, Stirling numbers of both kinds, and Eulerian numbers.

Theorem 2.2. For non-negative $n$ and $k$ we have

$$
\sum_{m=0}^{n}(-1)^{m}\left\{\begin{array}{c}
n \\
m
\end{array}\right\} \widehat{c}_{m, k}=(-1)^{n}(n+1)^{k} .
$$

Proof. The right-hand side is (besides the $(-1)$ factor) a poly-Cauchy permutation where the elements $\{\underline{1}, \ldots, \underline{n}\}$ are in increasing order (with some blocks of the elements $\{\overline{1}, \ldots, \bar{k}\}$ in between, before or after them), $\underline{\pi}^{n}$ is the identity permutation. We call such a permutation id-poly-Cauchy permutation.

We define an involution, $\phi$, to show that the left-hand side counts id-poly-Cauchy permutations of size $(n, k)$. The involution $\phi$ is defined on the set of poly-Cauchy permutations with an extra structure on the underlined (red) elements. Partition the elements $\{\underline{1}, \underline{2}, \ldots, \underline{n}\}$ into $m$ non-empty blocks (write the elements of a block in decreasing order, and consider the greatest element of a block as its "representative"). Then permute these blocks into a permutation with $j$ cycles, $j \leq m \leq n$. Insert between the cycles of blocks the overlined (blue) elements, $\{\overline{1}, \overline{2}, \ldots, \bar{k}\}$, in order to obtain a $(n, k)$-poly-Cauchy permutation with an extra structure on the substrings of underlined (red) elements (the blocks). Note that $j$ is the number of left-to-right maxima among the elements $\{\underline{1}, \underline{2}, \ldots, \underline{n}\}$.

For example, one set partition of $\{\underline{1}, \underline{2}, \ldots, \underline{9}\}$ into $m=5$ blocks is $B_{1}=\{\underline{4}, \underline{2}, \underline{1}\}, B_{2}=\{\underline{5}, \underline{3}\}, B_{3}=$ $\{\underline{6}\}, B_{4}=\{\underline{8}, \underline{7}\}, B_{5}=\{\underline{9}\}$. Now, we permute these five blocks to obtain a permutation in $j=3$ cycles. For example, the permutation $(32)(4)(51)$ corresponds to $\left(B_{3}, B_{2}\right)\left(B_{4}\right)\left(B_{5}, B_{1}\right)$. Finally, we insert the overlined (blue) blocks, for example we insert the blocks $\{\overline{1}, \overline{3}\},\{\overline{2}, \overline{4}, \overline{5}\},\{\overline{6}\}$, and obtain the $(9,6)$-poly-Cauchy permutation

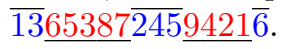

If the number of cycles is $n$, that is $j=n$, that can happen only one way, every block contains only one element and each cycle one block, hence, after inserting the blocks of $\{\overline{1}, \overline{2}, \ldots, \bar{k}\}$, we obtain an id-poly-Cauchy permutation.

Otherwise, i.e., if the number of cycles is smaller than $n$, then there is at least one cycle that contains at least two elements. Let $a$ be the greatest element that is not alone in a cycle.

If $a$ is in a block alone, merge it with the block that follows it in the cycle. If $a$ is not alone, separate it into a block to be alone.

This way we defined an involution, $\phi$, between the set of poly-Cauchy permutations with extra structure with an even number of left-to-right maxima in $\underline{\pi^{n}}$ and an odd number of left-to-right maxima in $\underline{\pi^{n}}$.

Many formulas given in the literature are consequences of Theorem 2.2 and modified forms of the involution $\phi$ can be used to provide combinatorial proofs for them. For instance, Komatsu presents a formula in [17] (Theorem 3) that can be seen as a generalization of Theorem 2.2. Let $\left\{\begin{array}{l}n \\ k\end{array}\right\}_{r}$ denote the $r$-Stirling numbers of the second kind that count for instance the number of partitions of $[n]$ into $k$ non-empty blocks such that $\{1,2, \ldots, r\}$ are in distinct blocks. These numbers were introduced by Broder [7]. 
Theorem 2.3 ([17, Theorem 3]). For non-negative $n, k$ and $r$, with $r \leq n$ we have

$$
\sum_{j=r}^{n}(-1)^{j}\left\{\begin{array}{l}
n \\
j
\end{array}\right\}_{r} \widehat{c}_{j, k}=\sum_{\ell=1}^{r}\left[\begin{array}{l}
r \\
\ell
\end{array}\right](n-r+\ell+1)^{k} .
$$

Proof. The idea of the proof is the same as in the proof of Theorem 2.2. Partition the elements $\{\underline{1}, \ldots, \underline{n}\}$ into $j$ non-empty blocks such that the elements $\{\underline{1}, \ldots, \underline{r}\}$ are in distinct blocks. As usual, we call these elements special elements and the remaining elements, $\{\underline{r+1}, \ldots, \underline{n}\}$, as non-special elements. Arrange the blocks into $i$ cycles $(i \leq j \leq n)$. Merge the so obtained permutation with blocks of $\{\overline{1}, \overline{2}, \ldots, \bar{k}\}$ in order to obtain a $(n, k)$ poly-Cauchy permutation with an extra structure on the substrings of underlined (red) elements (the blocks). As before, if the number of cycles is $n$, every block contains only one element, and each cycle one block, hence, after merging with the blocks of $\{\overline{1}, \overline{2}, \ldots, \bar{k}\}$ we obtain an id-poly-Cauchy permutation.

If $j \neq n$, there is at least one cycle that contains at least two elements. Note that the involution $\phi$, we defined in the proof of Theorem 2.2 can not be used in that form, since we can not merge blocks containing the special elements. We will slightly modify the definition of $a$, the base element of our involution, $\phi^{\prime}$. Let $a$ be the greatest non-special element which is not alone in a cycle.

If such an $a$ exists, we can apply the involution $\phi$ based on this element, i.e., if $a$ is in a block alone, merge it with the block that follows it in the cycle. If $a$ is not alone separate it into a block to be alone.

However, such an $a$ does not have to exist. If such an $a$ does not exist, it means, all the non-special elements are in distinct cycles, (and so distinct blocks), while the special elements are in distinct blocks by definition but not necessarily in distinct cycles. $\underline{\pi}^{n}$ is a permutation of the form: $\underline{\pi}^{n}=\left(c_{1}\right)\left(c_{2}\right) \cdots\left(c_{\ell}\right)(r+1)(r+2) \cdots(n)$, where $\ell$ is the number of cycles into the special elements are ordered. Note that these cycles contain only special elements (otherwise $a$ with the required property would exist). The overlined (blue) elements are inserted in the poly-Cauchy permutation into these cycles of $\pi^{n}$ (before or after).

The involution $\phi^{\prime}$ shows that the left-hand side of Identity (1) counts such poly-Cauchy permutations.

On the other hand, such poly-Cauchy permutations can be constructed by the following procedure. First, arrange the $r$ special elements into $\ell$ cycles, and take the non-special elements as fix points. Record this permutation in canonical cycle notation and insert the overlined (blue) elements $[k]$ in between, before or after the cycles decoding this insertion by a word $w=w_{1} w_{2} \cdots w_{k}$, where $w_{i}$ is the number of cycles to the left of the element $i$. The number of ways of doing this is clearly given by the formula of the right-hand side of the Identity (1).

Theorem 2.2 implies also the formula that connects poly-Cauchy numbers and poly-Bernoulli numbers [18]. The poly-Bernoulli numbers, denoted by $\mathbb{B}_{n}^{(k)}$, where $n$ is a positive integer and $k$ is an integer, are defined by the exponential generating function $[10]$

$$
\sum_{n=0}^{\infty} \mathbb{B}_{n}^{(k)} \frac{x^{n}}{n !}=\frac{\operatorname{Li}_{k}\left(1-e^{-x}\right)}{1-e^{-x}}
$$

where $\operatorname{Li}_{k}(z)=\sum_{i=1}^{\infty} \frac{z^{i}}{i^{k}}$ is the $k$-th polylogarithm function. Note that for $k=1$ we recover the Bernoulli numbers, that is, $\mathbb{B}_{n}^{(1)}=(-1)^{n} \mathbb{B}_{n}$, for $n \geq 0$, where $\mathbb{B}_{n}$ denotes the $n$-th Bernoulli number. Poly-Bernoulli numbers were introduced by Kaneko in 1997 [10] as he noticed that the generating function of the usual Bernoulli numbers can be generalized by using the polylogarithm function. From the combinatorial point of view, the array with negative $k$ indices are interesting since these numbers are integers (see sequence A099594 in $[22])$.

The poly-Bernoulli numbers with negative indices count several combinatorial objects (see for example $[1-6,21])$, in particular count Callan permutations [2]. An $(n, k)-$ Callan permutation is a permutation of $[n+k]$ such that each maximal contiguous substring whose support belongs to $\{1,2, \ldots, n\}$ (respectively $\{n+1, n+$ $2, \ldots, n+k\}$ ) is increasing (respectively decreasing). It is easy to see that an $(n, k)$-Callan permutation can be seen as an alternating sequence of blocks of partitions of $\{\underline{1}, \ldots, \underline{n}\}$ and $\{\overline{1}, \overline{2}, \ldots, \bar{k}\}$.

For non-negative $k$, the integral values, $\mathbb{B}_{n}^{(-k)}$, can be expressed in terms of $\widehat{c}_{n}^{(-k)}$ which can be proved combinatorially.

Theorem 2.4 ([18, Theorem 3.2]). For non-negative $n$ and $k$ we have

$$
\mathbb{B}_{n}^{(-k)}=(-1)^{n} \sum_{\ell=1}^{n} \sum_{m=1}^{n} m !\left\{\begin{array}{c}
n \\
m
\end{array}\right\}\left\{\begin{array}{c}
m \\
\ell
\end{array}\right\} \widehat{c}_{\ell}^{(-k)} .
$$

Proof. Let us rewrite the identity as follow:

$$
\mathbb{B}_{n}^{(-k)}=(-1)^{n} \sum_{m=1}^{n} m !\left\{\begin{array}{l}
n \\
m
\end{array}\right\} \sum_{\ell=1}^{m}(-1)^{\ell}\left\{\begin{array}{c}
m \\
\ell
\end{array}\right\} \widehat{c}_{\ell, k} .
$$


By Theorem 2.2 the inner sum equals to $(-1)^{m}(m+1)^{k}$, and we obtain the classical formula of poly-Bernoulli numbers:

$$
\mathbb{B}_{n}^{(-k)}=\sum_{m=1}^{n} m !\left\{\begin{array}{l}
n \\
m
\end{array}\right\}(-1)^{n+m}(m+1)^{k}
$$

Combinatorially, by the proof of the Theorem 2.2 after applying the involution, $\phi$, the inner sum

$$
\sum_{\ell=1}^{m}(-1)^{\ell}\left\{\begin{array}{c}
m \\
\ell
\end{array}\right\} \widehat{c}_{\ell, k}
$$

counts $(m, k)$-id-poly-Cauchy permutations. Now let $A_{m}$ be the set of Callan permutations with at most $m$ different underlined (red) blocks (blocks of elements $\{\underline{1}, \ldots, \underline{n}\})$. We obtain from an $(m, k)$-id-poly-Cauchy permutation an $(n, k)$-Callan permutation from $A_{m}$ as follows. Given an id-poly-Cauchy permutation, permute first the $m$ underlined (red) elements in $m$ ! ways. Then partition $\{\underline{1}, \ldots, \underline{n}\}$ into $m$ non-empty blocks, $\underline{B_{1}}, \underline{B_{2}}, \ldots, \underline{B_{m}}$, and replace the underlined (red) element $i$ in the poly-Cauchy permutation by a block $\underline{B_{i}}$. Applying the inclusion-exclusion principle completes the proof.

The other direction can be seen similarly combinatorially.

Theorem 2.5 ([18, Theorem 3.1]). For non-negative $n$ and $k$ we have

$$
\widehat{c}_{n}^{(-k)}=(-1)^{n} \sum_{\ell=1}^{n} \sum_{m=1}^{n} \frac{1}{m !}\left[\begin{array}{l}
n \\
m
\end{array}\right]\left[\begin{array}{c}
m \\
\ell
\end{array}\right] \mathbb{B}_{\ell}^{(-k)}
$$

Proof. Take now an $(\ell, k)$-Callan permutation and a permutation of $[m]$ into $\ell$ cycles. Replace each underlined (red) element in the Callan permutation by a cycle. If there are more cycles in a block, take the product of the cycles and record this way $m$ elements into the overlined (blue) blocks of the Callan permutation. (So, the $m$ elements are not in cycles anymore, but create subsequences of a permutation of $m$ with places in between.) (Note that if we ignore the overlined (blue) blocks, the underlined (red) elements determine a permutation of $m$, and summing up on all $\ell$, every permutation will occur.) Order the elements $\{\underline{1}, \underline{2}, \ldots, \underline{n}\}$ into $m$ cycles and replace each underlined (red) element in our construction by a cycle in the canonical order (first the cycle with the least greatest element and so on). This way we obtain a poly-Cauchy permutation.

Example 2.7. We show the proof process with an example. Let $n=9, m=5, \ell=3, k=6$. Let the $(\ell, k)$ Callan permutation be $\overline{6} 23 \overline{135} \underline{1} \overline{24}$, the permutation of $[\mathrm{m}]$ into $\ell$ cycles $(1)(43)(52)$, and the permutation of $[n]$ into $m$ cycles $(43)(5)(71)(82)(96)$.

$$
\begin{aligned}
& \overline{6} 2 \underline{135} \underline{124} \rightarrow \overline{6}(43)(52) \overline{135}(1) \overline{24} \quad \rightarrow \quad \overline{6} \underline{5432} \overline{135} \underline{124}
\end{aligned}
$$

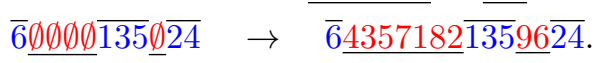

Next we provide a combinatorial proof for the closed formula of poly-Cauchy numbers.

Theorem 2.6. For non-negative $n$ and $k$ we have

$$
\widehat{c}_{n, k}=\sum_{j=0}^{k} j !\left[\begin{array}{c}
n+1 \\
j+1
\end{array}\right]\left\{\begin{array}{l}
k+1 \\
j+1
\end{array}\right\} .
$$

Proof. We construct the $(n, k)$-poly-Cauchy permutations as follows. Let $\bar{K}$ be the set $[k]$ extended by a special element $\bar{*}$. First, partition the set $\bar{K}$ into $j+1$ non-empty blocks. Second, order the set $[n]$ into $\ell$ cycles in the canonical cycle notation, with $j \leq \ell \leq n$. Then we choose $j$ of these cycles to insert an overlined (blue) ordinary block (blocks without the element $\bar{*}$ ) directly before each cycle, so we have $\ell(\ell-1) \cdots(\ell-j+1)=\left(\begin{array}{l}\ell \\ j\end{array}\right) j$ ! options to insert and organize these blocks. Finally, the extra block, that contains $\bar{*}$ is inserted after the last underlined (red) cycle, at the end of the arrangement, and we delete the element $\bar{*}$. So if the extra block contains only $\bar{*}$ our poly-Cauchy permutation ends with an underlined (red) element; if it contains also other elements, it ends with an overlined (blue) element. Therefore, we have

$$
\widehat{c}_{n, k}=\sum_{j=0}^{k}\left\{\begin{array}{l}
k+1 \\
j+1
\end{array}\right\} \sum_{\ell=j}^{n}\left[\begin{array}{l}
n \\
\ell
\end{array}\right]\left(\begin{array}{l}
\ell \\
j
\end{array}\right) j !=\sum_{j=0}^{k} j !\left\{\begin{array}{l}
k+1 \\
j+1
\end{array}\right\}\left[\begin{array}{c}
n+1 \\
j+1
\end{array}\right] .
$$

Notice that in the last equality we use the identity $\sum_{\ell=j}^{n}\left(\begin{array}{l}\ell \\ j\end{array}\right)\left[\begin{array}{l}n \\ \ell\end{array}\right]=\left[\begin{array}{c}n+1 \\ j+1\end{array}\right]$. 
In the following two theorems we give recurrence relations to calculate the sequence $\widehat{c}_{n, k}$.

Theorem 2.7. For $n \geq 1$ and $k \geq 0$ we have

$$
\widehat{c}_{n, k}=(n-1) \widehat{c}_{n-1, k}+\sum_{i=0}^{k}\left(\begin{array}{c}
k \\
i
\end{array}\right) \widehat{c}_{n-1, k-i}
$$

Proof. The greatest underlined (red) element, $n$, is surely in the last cycle of the permutation $\underline{\pi^{n}}$, but we consider the cases when it is the very last underlined (red) element and when it is followed by some other underlined (red) elements separately. First, assume that $n$ is the last underlined (red) element. An $(n, k)$-poly-Cauchy permutation can be obtained in the following way. First, we choose $i$ overlined (blue) elements ( $i$ can be empty) in a block, say $\bar{B}$. We take a $(n-1, k-i)$-poly-Cauchy permutation, and extend it by the sequence of $\underline{n} \bar{B}$ at the end. This can be done in $\sum_{i=0}^{k}\left(\begin{array}{c}k \\ i\end{array}\right) \widehat{c}_{n-1, k-i}$ ways.

Next, assume that $n$ is not the last underlined (red) element. In order to show that the number of such $(n, k)$-poly-Cauchy permutations is $(n-1) \widehat{c}_{n-1, k}$, we establish a bijection. Given an $(n-1, k)$-poly-Cauchy permutation $\pi$, and a value $1 \leq i \leq n-1$, we construct an $(n, k)$-poly-Cauchy permutation $\pi^{\prime}$ as follows. $\pi$ is a merging of $\frac{\pi^{n-1}}{}$ and $\overline{\pi^{k}}$. Consider $\frac{\pi^{n-1}}{\pi^{n}}$ as the product of cycles. Insert $n$ before the element $i$ in its cycle, to obtain $\underline{\pi^{\prime} n}$. Merge now $\frac{\pi^{\prime} n}{}$ and $\overline{\pi^{k}}$ again together. Note that we did not change the places where the overlined (blue) elements are inserted, but we possibly changed the relative order of the underlined (red) cycles, since the cycle containing $n$ may be shifted to the end, and other cycles may be shifted to the left by one. Since each step can be reversed, this procedure is a bijection, which implies our statement.

Example 2.8. We give an example for making the bijection in the previous proof clearer. Let $\pi=\overline{6} \underline{43} \overline{24} \underline{561} \overline{135}$ $\underline{827}$ and $i=6$. The cycles of $\underline{\pi^{8}}$ are $(\underline{43}),(\underline{5}),(\underline{61})$ and $(\underline{827})$. We insert $\underline{9}$ after $\underline{6}$ and obtain $(\underline{61}) \rightarrow(\underline{691})=$ (916). After merging the cycles and blocks again, we obtain $\underline{\pi^{\prime}}=\overline{6} \underline{43} \overline{24} \underline{5827} \overline{135} \underline{916}$.

Theorem 2.8. For $n \geq 1$ and $k \geq 0$ we have

$$
\widehat{c}_{n, k}=\sum_{i=0}^{n} \sum_{j=0}^{k}(n-1)_{i}\left(\begin{array}{c}
k \\
j
\end{array}\right) \widehat{c}_{n-1-i, k-j} .
$$

Proof. Let $j$ be the number of the last overlined (blue) block (possibly empty), and $i$ be the number of elements that the last underlined (red) cycle contains besides the element $n$. (In the canonical cycle notation the last cycle necessarily contains $n$.) We choose the elements for the block in $\left(\begin{array}{c}k \\ j\end{array}\right)$ ways and construct the last cycle by arranging $i$ elements after $n$ in $\left(\begin{array}{c}n-1 \\ i\end{array}\right) i !=(n-1)_{i}$ ways. From the remaining elements, we construct a poly-Cauchy permutation.

The Eulerian numbers $\left\langle\begin{array}{c}n \\ k\end{array}\right\rangle$ count the number of permutations $\pi=\pi_{1} \pi_{2} \cdots \pi_{n}$ with $k-1$ descents, that is $k-1=\left|\left\{i \in[n-1]: \pi_{i}>\pi_{i+1}\right\}\right|$. Note that a permutation $\pi$ of $[n]$ with $k-1$ descents is the union of $k$ increasing subsequences of consecutive entries, also called ascending runs. For example, if $\pi=258193647$, then 3,5 , and 7 are the descents. Moreover, $\pi$ is the union of the ascending runs: $258,19,36$, and 47 . In Theorem 2.9 we give a combinatorial identity of the sequence $\widehat{c}_{n, k}$ involving the Eulerian numbers.

Theorem 2.9. For non-negative $n$ and $k$ we have

$$
\widehat{c}_{n, k}=\sum_{m=0}^{n} \sum_{i=0}^{m}\left(\begin{array}{c}
k-i \\
m-i
\end{array}\right)\left\langle\begin{array}{l}
k \\
i
\end{array}\right\rangle\left[\begin{array}{l}
n+1 \\
m+1
\end{array}\right] .
$$

Proof. We turn now our point of view a little over and consider the construction of a poly-Cauchy permutation as inserting underlined (red) cycles into a permutation of overlined (blue) elements $\bar{\pi}^{k}$. The properties of polyCauchy permutations require that the ascending runs of $\pi^{k}$ are separated by an underlined (red) cycle. But it is not forbidden to insert cycles after an ascent, cutting ascending runs this way into more pieces. Hence, the $(n, k)$-poly-Cauchy permutations can be constructed by the following process. Take a permutation of $[k]$ with $i$ ascending runs and mark $m-i$ ascents. Permute the underlined (red) elements into at least $m$ cycles, say $\ell$, and choose $m$ out of them. Merge the two permutations in order to obtain a poly-Cauchy permutation by inserting a chosen cycle after each ascending run and marked ascent.

\section{Poly-Cauchy polynomials of the second kind}

This section introduces a combinatorial interpretation for the poly-Cauchy polynomials of the second kind. The poly-Cauchy polynomials of the second kind, denoted by $\widehat{c}_{n}^{k}(z)$, were introduced by Kamano and Komatsu [9] 
by using the following analytical formula

$$
\widehat{c}_{n}^{(k)}(z):=n ! \underbrace{\int_{0}^{1} \ldots \int_{0}^{1}}_{k}\left(\begin{array}{c}
-x_{1} x_{2} \cdots x_{k}-z \\
n
\end{array}\right) d x_{1} d x_{2} \cdots d x_{k} .
$$

The above definition is equivalent to the explicit formula

$$
\widehat{c}_{n}^{k}(z)=(-1)^{n} \sum_{m=0}^{n}\left[\begin{array}{c}
n \\
m
\end{array}\right] \sum_{i=0}^{m}\left(\begin{array}{c}
m \\
i
\end{array}\right) \frac{(-z)^{i}}{(m-i+1)^{k}} .
$$
denote

We consider poly-Cauchy polynomials of the second kind with negative $k$-indices. More precisely, let us

$$
\widehat{c}_{n, k}(z):=\sum_{m=0}^{n}\left[\begin{array}{c}
n \\
m
\end{array}\right] \sum_{i=0}^{m}\left(\begin{array}{c}
m \\
i
\end{array}\right)(-1)^{i}(m-i+1)^{k} z^{i} .
$$

We define partial poly-Cauchy permutations as a pair $P=(\pi, \sigma)$, where the reduction of $\pi$ on the underlined (red) elements is a partial permutation of $[n]$ (not all elements of $[n]$ are included) and $\sigma$ is a permutation of the remaining elements of $[n]$. Further, we define the weight of a partial poly-Cauchy permutation, denoted by $w(P)=w(\pi, \sigma)$, as the number of cycles in the permutation $\sigma$.

Example 3.1. The weight of the partial poly-Cauchy permutation $(\overline{24} \underline{61} \overline{6} \underline{78} \overline{135} \underline{93},(\underline{24})(\underline{5}))$ is 2 .

Let $\mathcal{P} \mathcal{P}_{n, k}$ denote the set of partial poly-Cauchy permutations.

Theorem 3.1. We have

$$
\sum_{P \in \mathcal{P P}_{n, k}}(-z)^{w(P)}=\widehat{c}_{n, k}(z)
$$

Proof. The sum is clearly equal to the formula (4) according to our previous considerations.

Recall the well-known relation between the falling factorial $(x)_{n}=x(x-1) \cdots(x-n+1)$ and the (signed) Stirling number of the first kind, $s(n, k)$ :

$$
(x)_{n}=\sum_{k=0}^{n} s(n, k) x^{k} .
$$

From the combinatorial point of view this identity expresses that if we weight a permutation $\pi$ with the number of its cycles, i.e., $w(\pi)=\#$ of cycles, we have

$$
(-1)^{n}(x)_{n}=\sum_{\pi \in S_{n}}(-x)^{w(\pi)}
$$

where $S_{n}$ denotes as usual the set of permutations of $[n]$. The following theorem is based on this classical result.

Theorem 3.2 ([11, Theorem 7]). For non-negative $n$ and $k$ we have

$$
\widehat{c}_{n}^{(-k)}(z)=\sum_{m=0}^{n}\left(\begin{array}{l}
n \\
m
\end{array}\right) \widehat{c}_{n-m}^{(-k)}(z)_{m}
$$

Proof. Let $m$ be the number of elements that are contained in $\sigma$ in the partial poly-Cauchy permutation $P=(\pi, \sigma)$. Choose these $m$ elements in $\left(\begin{array}{c}n \\ m\end{array}\right)$ ways and construct $\pi$ from the remaining elements in $\widehat{c}_{n-m, k}$ ways. Hence, we have

$$
\sum_{P \in \mathcal{P} \mathcal{P}_{n, k}}(-z)^{w(P)}=\sum_{m=0}^{n}\left(\begin{array}{l}
n \\
m
\end{array}\right) \widehat{c}_{n-m, k}(-1)^{m}(z)_{m} .
$$

Since $\widehat{c}_{n-m, k}=(-1)^{n-m} \widehat{c}_{n-m}^{(-k)}$, the theorem follows.

The theorems proven in the previous section can be generalized easily to partial poly-Cauchy permutations, and hence to poly-Cauchy polynomials. As an example, we present the combinatorial proof for the generalization of Theorem 2.2. 
Theorem 3.3 ([9, Theorem 4]). For non-negative $n$ and $k$ we have

$$
\sum_{m=0}^{n}(-1)^{m}\left\{\begin{array}{c}
n \\
m
\end{array}\right\} \widehat{c}_{m, k}(z)=\sum_{i=0}^{n}\left(\begin{array}{c}
n \\
i
\end{array}\right)(-1)^{i} z^{i}(n-i+1)^{k} .
$$

Proof. Applying the involution $\phi$ of the proof of Theorem 2.2 on the set of partial poly-Cauchy permutations $\mathcal{P} \mathcal{P}$, we recognize that only such partial poly-Cauchy permutations do not cancel in that the underlined (red) elements are in increasing order. This means now that $\sigma$ is also fixed as an identity permutation on the elements that are contained there. So the weight of $\sigma$ is simply $(-z)^{i}$, if $i$ is the size of $\sigma$.

We show combinatorially two identities that are typical for polynomials. Moreover, in these proofs, we introduce another combinatorial interpretation of the poly-Cauchy polynomials (which arises also very naturally). We omit the proofs of the other generalizations as they are straightforward.

Looking at the formula (5), we can give a combinatorial interpretation for $z$ positive integers as follow. Consider now a poly-Cauchy permutation with $z$ ordered boxes. Choose some elements of $[n]$ and put them into one of the boxes and form from the remaining elements a poly-Cauchy permutation. We call such a construction an extended poly-Cauchy permutation. Having this picture in mind, the following two identities (6) and (7) are immediate.

Theorem 3.4 ([11]). We have

$$
\widehat{c}_{n, k}(z+1)=\widehat{c}_{n, k}(z)+n \widehat{c}_{n-1, k}(z)
$$

Proof. The left-hand side counts extended poly-Cauchy permutations with $z+1$ boxes. There are two cases, the last box contains an element, or not. If the last box is empty, we can delete it and the number of such extended permutations is just $\widehat{c}_{n, k}(z)$. If the last box contains any of the $n$ elements (that can be chosen in $n$ ways) the remaining $n-1$ elements create with the $k$ elements an extended poly-Cauchy permutation with $z$ boxes.

Theorem 3.5 ([11, Eq. (33)]). We have

$$
\widehat{c}_{n, k}(z+y)=\sum_{j=0}^{n}\left(\begin{array}{l}
n \\
j
\end{array}\right) \widehat{c}_{j, k}(z)(y)_{n-j} .
$$

Proof. The left-hand side counts extended poly-Cauchy permutations with $z+y$ boxes. We obtain such an object by choosing $j$ elements out of $[n]$ from that we construct an extended poly-Cauchy permutation with $z$ boxes, and we put the remaining $n-j$ elements into the remaining $y$ boxes. This construction corresponds to the right-hand side.

\section{Other generalizations}

In this section, we recall some of the generalizations of poly-Cauchy numbers and possible extensions of our combinatorial model in these directions.

\subsection{Shifted poly-Cauchy numbers}

Let $\alpha$ be a positive real number. The shifted poly-Cauchy numbers of the second kind have the explicit formula $[19]$

$$
\widehat{c}_{n, \alpha}^{k}:=(-1)^{n} \sum_{m=0}^{n}\left[\begin{array}{l}
n \\
m
\end{array}\right] \frac{1}{(m+\alpha)^{k}} .
$$

Let us define the objects augmented poly-Cauchy permutations as a poly-Cauchy permutations of the second kind ending with an underlined (red) element and augmented with (possibly empty) $\alpha$ boxes with overlined (blue) elements. (The order of the overlined (blue) elements in the boxes are not important, however, the boxes are distinguishable.) An example with $\alpha=3$ is given below. The boxes are indicated with $\alpha-1$ bars.

$$
\overline{49} \underline{612} \overline{6} \underline{748} \overline{13} \underline{953} \overline{27} \| \overline{58} .
$$

Theorem 4.1. The $(n, k)$-augmented poly-Cauchy permutations with $\alpha$ boxes are enumerated by the shifted poly-Cauchy numbers, $\widehat{c}_{n, k, \alpha}=\sum_{m=0}^{n}\left[\begin{array}{c}n \\ m\end{array}\right](m+\alpha)^{k}$. 
Proof. The difference to the base case is that the possible places where overlined (blue) elements can be inserted are augmented by $\alpha-1$ bars. In a poly-Cauchy permutation overlined (blue) elements can be inserted between (before or after) underlined (red) cycles. Hence, there are now $m+\alpha$ possibilities, when we consider an underlined (red) permutation with $m$ cycles.

A closed formula of the shifted poly-Cauchy numbers, the generalization of Theorem 2.6, can be shown combinatorially as follow.

Theorem 4.2 ([19, Theorem 10]). For $n, k$, and $\alpha$ non-negative integers we have

$$
\widehat{c}_{n, k, \alpha}=\sum_{i=0}^{k} \sum_{j=0}^{i} j !\left[\begin{array}{c}
n+1 \\
j+1
\end{array}\right]\left(\begin{array}{c}
k \\
i
\end{array}\right)\left\{\begin{array}{l}
i \\
j
\end{array}\right\} \alpha^{k-i} .
$$

Proof. Let $k-i$ be the number of overlined (blue) elements in the boxes. We choose these elements in $\left(\begin{array}{l}k \\ i\end{array}\right)$ ways and put them into the boxes in $\alpha^{k-i}$ ways. To the left of the boxes there is a poly-Cauchy permutation on the underlined (red) elements $[n]$, and overlined (blue) elements $[i]$, such that the last element is underlined (red). The argument of Theorem 2.6 has to be slightly modified to take the type of the last element into account. Namely, we do not need the extra block, so we do not extend the overlined (blue) element set now with $\bar{*}$. We take a partition of the $i$ overlined (blue) elements into $j$ blocks in $\left\{\begin{array}{l}i \\ j\end{array}\right\}$ ways and insert each block directly before the chosen cycle.

\subsection{Incomplete poly-Cauchy numbers of the second kind}

The incomplete poly-Cauchy numbers of the second kind have an explicit formula [16]

$$
\widehat{c}_{n, \leq m}^{k}:=(-1)^{n} \sum_{i=0}^{n}\left[\begin{array}{l}
n \\
i
\end{array}\right]_{\leq m} \frac{1}{(i+1)^{k}},
$$

where $\left[\begin{array}{l}n \\ k\end{array}\right]_{\leq m}$ denotes the restricted Stirling numbers of the first kind. This sequence counts the number of permutations on $n$ elements with $k$ cycles such that each cycle contains at most $m$ items. From a similar argument we have that $\widehat{c}_{n, k, \leq m}=\sum_{i=0}^{n}\left[\begin{array}{l}n \\ i\end{array}\right]_{\leq m}(i+1)^{k}$ counts the number of poly-Cauchy permutations of $[n+k]$, such that the size of each cycle in the cycle decomposition of the permutation whose support belongs to $\{1,2, \ldots, n\}$ is at most $m$.

Example 4.1. For $n=3$ and $k=1, m=2$ we have the following restricted poly-Cauchy permutations:

$\underline{123 \overline{1}}, \quad \underline{12} \overline{1} \underline{3}, \quad \underline{132} \overline{1}, \quad \underline{1} \overline{1} \underline{23}, \quad \underline{1} \overline{1} \underline{32}, \quad \underline{213} \overline{1}, \quad \underline{21} \overline{1} \underline{3}$

$\underline{231 \overline{1}}, \quad \underline{2} \overline{1} \underline{1}, \quad \overline{1} 123, \quad \overline{1} \underline{132}, \quad \overline{1} \underline{213}, \quad \overline{1} \underline{231}$.

So, $\widehat{c}_{3,1, \leq 2}=13$.

The associated poly-Cauchy numbers of the second kind have an explicit formula

$$
\widehat{c}_{n, \leq m}^{k}:=(-1)^{n} \sum_{i=0}^{n}\left[\begin{array}{l}
n \\
i
\end{array}\right]_{\geq m} \frac{1}{(i+1)^{k}},
$$

where $\left[\begin{array}{l}n \\ k\end{array}\right]_{\geq m}$ denotes the associated Stirling numbers of the first kind. This sequence counts the number of permutations on $n$ elements with $k$ cycles with the restriction that each cycle contains at least $m$ items. So, the sequence $\widehat{c}_{n, k, \geq m}:=\sum_{i=0}^{n}\left[\begin{array}{l}n \\ i\end{array}\right]_{\geq m}(i+1)^{k}$ counts the number of poly-Cauchy permutations of $[n+k]$, such that the size of each cycle in the cycle decomposition of the permutation whose support belongs to $\{1,2, \ldots, n\}$ is at least $m$.

Example 4.2. For $n=3$ and $k=1, m=2$ we have the permutations

$$
\underline{312} \overline{1}, \quad \underline{321} \overline{1}, \quad \overline{1} 312, \quad \overline{1} 321 .
$$

So, $\widehat{c}_{3,1, \geq 2}=4$. 


\section{$4.3 q$-poly-Cauchy numbers}

The $q$-poly-Cauchy numbers of the second kind have an explicit formula [13]

$$
\widehat{c}_{n, q}^{(k)}:=(-1)^{n} \sum_{m=0}^{n}\left[\begin{array}{l}
n \\
m
\end{array}\right] \frac{q^{n-m}}{(m+1)^{k}} .
$$

It is clear that the sequence $\widehat{c}_{n, k}(q):=\sum_{m=0}^{n}\left[\begin{array}{c}n \\ m\end{array}\right] q^{n-m}(m+1)^{k}$ counts the number of poly-Cauchy permutations of $[n+k], \pi$, such that the non left-to-right maxima of $\underline{\pi}^{n}$ are colored by one of $q$ colors.

Example 4.3. For $n=2$ and $k=2$ we have the permutations

$$
\begin{aligned}
& \overline{12} \underline{12} \rightarrow 1, \quad \overline{12} \underline{21} \rightarrow q, \quad \overline{1} \underline{12} \overline{2} \rightarrow 1, \quad \overline{1} \underline{21} \overline{2} \rightarrow q, \quad \overline{2} \underline{12} \overline{1} \rightarrow 1, \quad \overline{2} \underline{1} 1 \overline{1} \rightarrow q, \quad \overline{1} \underline{1} \overline{2} \underline{2} \rightarrow 1 \\
& \overline{2} \underline{1} \overline{1} \underline{2} \rightarrow 1, \quad \underline{12} \overline{12} \rightarrow 1, \quad \underline{21} \overline{12} \rightarrow q, \quad \underline{1} \overline{12} \underline{2} \rightarrow 1, \quad \underline{1} \overline{1} \underline{2} \overline{2} \rightarrow 1, \quad \underline{1} \overline{2} \underline{2} \overline{1} \rightarrow 1 .
\end{aligned}
$$

So, $\widehat{c}_{2,2}(q)=4 q+9$.

\section{$4.4 q$-poly-Cauchy permutations with a parameter}

There is another generalization, $q$-poly-Cauchy numbers with a parameter [15], given by the explicit formula

$$
\widehat{c}_{n, \rho, q}^{(k)}=(-1)^{n} \sum_{m=0}^{n}\left[\begin{array}{c}
n \\
m
\end{array}\right] \frac{\rho^{n-m}}{[m+1]_{q}^{k}},
$$

whith $[n]_{q}=1+q+\cdots+q^{n-1}$. The expression with negative $k$ and ignoring the sign, defined as $\widehat{c}_{n, k, \rho, q}=$ $\sum_{m=0}^{n}\left[\begin{array}{c}n \\ m\end{array}\right] \rho^{n-m}[m+1]_{q}^{k}$, counts poly-Cauchy permutations with some weight on both the underlined (red) elements and overlined (blue) elements. Given a poly-Cauchy permutation $\pi$, as in the case of $q$-poly-Cauchy permutations we can color the non left-to-right maxima of $\underline{\pi^{n}}$ by one of $\rho$ colors. This notion is extended by

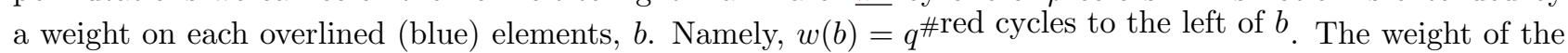
poly-Cauchy permutation $\pi$ is the product of weights of all its elements. Formally, let $\mathcal{P}_{n, k}$ denote the set of poly-Cauchy permutations of $[n+k]$, and $\mu(\pi)$ the number of non left-to-right maxima in $\underline{\pi^{n}}$. Then we have

$$
\widehat{c}_{n, k, \rho, q}=\sum_{\pi \in \mathcal{P}} \rho^{\mu(\pi)} \prod_{i=1}^{k} q^{w(n+i)} .
$$

Example 4.4. For $n=2$ and $k=2$ the weight of the permutations are

$$
\begin{aligned}
& \overline{12} \underline{12} \rightarrow 1, \quad \overline{12} \underline{21} \rightarrow \rho, \quad \overline{1} \underline{12} \overline{2} \rightarrow q^{2}, \quad \overline{1} \underline{21} \overline{2} \rightarrow q \rho, \quad \overline{2} \underline{12} \overline{1} \rightarrow q^{2}, \quad \overline{2} \underline{1} 1 \overline{1} \rightarrow q \rho, \quad \overline{1} \underline{1} \overline{2} \underline{2} \rightarrow q \\
& \overline{2} \underline{1} \overline{1} \underline{2} \rightarrow q, \quad \underline{12} \overline{12} \rightarrow q^{4}, \quad \underline{21} \overline{12} \rightarrow q^{2} \rho, \quad \underline{1} \overline{12} \underline{2} \rightarrow q^{2}, \quad \underline{1} \overline{1} \underline{2} \overline{2} \rightarrow q^{3}, \quad \underline{1} \overline{2} \underline{2} \overline{1} \rightarrow q^{3} .
\end{aligned}
$$

\section{Conclusion}

In this paper, we presented a combinatorial interpretation of poly-Cauchy numbers of the second kind. We showed how this model can be extended to some of the generalizations that were introduced in the literature on these numbers. However, this paper on this topic is not even aimed to be complete. One of our goals was to show the power of combinatorial thinking, proofs with pictures.

In this section, we list some open questions that would be interesting to understand combinatorially.

1. We studied in this work only the poly-Cauchy numbers (and their generalizations) of the second kind. The question arises naturally; is it possible to give a similar interpretation of the poly-Cauchy of the first kind? The relation between the two kinds of poly-Cauchy numbers is given for instance by the following formula:

$$
(-1)^{n} \frac{c_{n}^{k}}{n !}=\sum_{m=1}^{n}\left(\begin{array}{c}
n-1 \\
m-1
\end{array}\right) \frac{\hat{c}_{m}^{k}}{m !} .
$$

2. We did not present a combinatorial proof for all of the formulas that are known, though we think most of the identities could be attacked using our combinatorial interpretation. The interested reader can try to find combinatorial proof for them. For example, recently, Komatsu [17] (Theorem 8) showed an annihilation formula

$$
\sum_{\ell=0}^{k}\left\{\begin{array}{c}
n+1 \\
n-\ell+1
\end{array}\right\}_{n-k} \widehat{c}_{n-\ell}^{(-k)}=0 .
$$


3. There are also several interesting identities involving generalizations of the poly-Cauchy numbers that have a combinatorial "flavour". As an example, we recall the expression that was given by Komatsu and Szalay [19] in their study about the shifted poly-Cauchy numbers:

$$
\widehat{c}_{n, \alpha}^{(-k)}=(-1)^{\alpha-1} \sum_{\mu=0}^{\alpha-1} Q_{\mu}(n, \alpha) \widehat{c}_{n+\mu}^{(-k)}
$$

where

$$
Q_{\mu}(n, \alpha)=\sum_{i=0}^{\alpha-\mu-1}\left(\begin{array}{c}
\alpha-1 \\
i
\end{array}\right)\left\{\begin{array}{c}
\alpha-i-1 \\
\mu
\end{array}\right\} n^{i}, \quad(0 \leq \mu \leq \alpha-1) .
$$

4. One of the interesting far reaching generalizations of poly-Cauchy numbers are q-multiparameter-polyCauchy polynomials that were originally defined by Komatsu and Szalay [20] using Jackson's $q$-integrals. We recall here the explicit form of $q$-multiparameter-poly-Cauchy polynomials of the second kind derived in [20] that may be treated combinatorially. For $n$ and $k$ integers, $(n \geq 0)$ let $L=\left(\ell_{1}, \ldots, \ell_{k}\right)$ be a $k$-tuple of real numbers with $\ell=\ell_{1} \cdots \ell_{k} \neq 0$ and $A=\left(\alpha_{0}, \alpha_{1}, \ldots, \alpha_{n-1}\right)$ an $n$-tuple of real numbers. Let $q$ be a real number with $0<q<1$. The multiparameter Stirling number of the first kind, $S_{1}(n, k, A)$ is defined by the equation

$$
\left(t-\alpha_{0}\right)\left(t-\alpha_{1}\right) \cdots\left(t-\alpha_{n-1}\right)=\sum_{m=0}^{n} S_{1}(n, m, A) t^{m} .
$$

The $q$-multiparameter-poly-Cauchy polynomials of the second kind has an explicit form [20, Theorem 2]

$$
\widehat{c}_{n, L, A, q}^{(k)}(z)=\sum_{m=0}^{n}(-1)^{m} S_{1}(n, m, A) \sum_{i=0}^{m}\left(\begin{array}{c}
m \\
i
\end{array}\right) \frac{(-z)^{i} \ell^{m-i+1}}{[m-i+1]_{q}^{k}} .
$$

Can we give any combinatorial meaning to these polynomials?

5. There are even more generalizations in the literature, as multi-poly-Cauchy numbers and polynomials (cf. [12]) that we do not recall here explicitly, but would like to turn the attention of the reader to the wealth of the topic.

6. The On-Line Encyclopedia of Integer Sequences [22] lists 30 sequences for poly-Cauchy numbers. Different from poly-Bernoulli numbers, none of the sequences arising from poly-Cauchy numbers has had any meaningful combinatorial interpretation.

\section{Acknowledgements}

The authors would like to thank the anonymous referee for carefully reading the paper and giving helpful comments and suggestions. The second author was partially supported by Universidad Nacional de Colombia, Project No. 46240.

\section{References}

[1] B. Bényi, Advances in bijective combinatorics, PhD thesis, (2014), available electronically at http://www . math.u-szeged.hu/phd/dreposit/phdtheses/benyi-beata-d.pdf.

[2] B. Bényi and P. Hajnal, Combinatorics of poly-Bernoulli numbers, Studia Sci. Math. Hungarica 52 (2015), 537-558.

[3] B. Bényi and P. Hajnal, Combinatorial properties of poly-Bernoulli relatives, Integers 17 (2017), \#A31, $1-26$.

[4] B. Bényi and G. V. Nagy, Bijective enumerations of $\Gamma$-free matrices, Adv. in Appl. Math. 96 (2018), 195-215.

[5] B. Bényi and J. L. Ramírez, On q-poly-Bernoulli numbers arising from combinatorial interpretations, arXiv:1909.09949.

[6] C. Brewbaker, A combinatorial interpretation of the poly-Bernoulli numbers and two Fermat analogues, Integers 8 (2008), \#A02, 1-9. 
[7] A. Z. Broder, The r-Stirling numbers, Disc. Math. 49 (1984), 241-259.

[8] M. Cenkci and P. T. Young, Generalizations of poly-Bernoulli and poly-Cauchy numbers, Eur. J. Math. 1 (5) (2015), 799-828.

[9] K. Kamano and T. Komatsu, Poly-Cauchy polynomials, Mosc. J. Comb. Number Theory 3 (2013), 61-87.

[10] M. Kaneko, Poly-Bernoulli numbers, J. Théor. Nombres Bordeaux 9 (1997), 221-228.

[11] D. S. Kim and T. Kim, Poly-Cauchy numbers and polynomials of the second kind, Adv. Differ. Equ. 36 (2014).

[12] D. S. Kim, T. Kim, T. Komatsu, and S.-H Rim, Multi-poly-Cauchy polynomials, J. Comput. Anal. Appl. 18 (2015), 536-550.

[13] T. Komatsu, Poly-Cauchy numbers with a q parameter, Ramanujan J. 31 (2013), 353-371.

[14] T. Komatsu, Poly-Cauchy numbers, Kyushu J. Math. 67 (2013), 143-153.

[15] T. Komatsu, q-poly-Bernoulli numbers and q-poly-Cauchy numbers with a parameter by Jackson's integrals, Indag. Math. 27 (2016), 100-111.

[16] T. Komatsu, Incomplete poly-Cauchy numbers, Monatsh Math. 180 (2016), 271-288.

[17] T. Komatsu, Recurrence relations of poly-Cauchy numbers by the r-Stirling transform, arXiv:2103.15291.

[18] T. Komatsu and F. Luca, Some relationships between poly-Cauchy numbers and poly-Bernoulli numbers, Ann. Math. Inform. 41 (2013), 99-105.

[19] T. Komatsu and L. Szalay, Shifted poly-Cauchy numbers, Lith. Math. J. 54 (2) (2014), $166-181$.

[20] T. Komatsu and L. Szalay, q-multiparameter-Bernoulli polynomials and q-multiparameter-Cauchy polynomials by Jackson's integrals, Integers 16 (2016), \#A39, 1-11.

[21] T. Matsusaka, Symmetrized poly-Bernoulli numbers and combinatorics, J. Integer Seq. 23 (2020), Article 20.9.2.

[22] N. J. A. Sloane, The On-Line Encyclopedia of Integer Sequences, available electronically at http://oeis. org/. 